MATEC Web of Conferences 52, 03001 (2016)

DOI: $10.1051 /$ matecconf $/ 20165203001$

(C) Owned by the authors, published by EDP Sciences, 2016

\title{
A Tooth Flank Crowning Method by Applying A Novel Crossed Angle Function Between the Hob Cutter and Work Gear in The Gear Hobbing Process
}

\author{
Yu-Ren $\mathrm{Wu}^{1, \mathrm{a}}$, Van-The $\mathrm{Tran}^{2}$ and Ruei-Hung $\mathrm{Hsu}^{3}$ \\ ${ }^{1}$ Department of Mechanical Engineering, National Central University, 300, Jhongda Rd., Jungli City, Taoyuan 320, \\ Taiwan \\ ${ }^{2}$ Department of Mechanical Engineering, Hung Yen University of Technology and Education, 5 Rd., Hung Yen City, \\ Vietnam \\ ${ }^{3}$ Bachelor's Program in Precision System Design, Feng Chia University, 100 Wenhwa Rd., Seatwen, Taichung \\ 40724, Taiwan
}

\begin{abstract}
In this paper, a novel longitudinal tooth flank crowning method is proposed by setting the crossed angle between the hob cutter and work gear as a linear function of hob's traverse feed movement in the gear hobbing process. However, this method makes twisted tooth flanks on the hobbed work gear. Therefore, a variable pressure angle hob cutter is applied to obtain an anti-twist tooth flank of hobbed work gear. A computer simulation example is performed to verify the superiority of the proposed novel hobbing method by comparing topographies of the crowned work gear surfaces hobbed by a standard hob cutter and a variable pressure angle hob cutter.
\end{abstract}

\section{Introduction}

In the conventional gear hobbing process, the longitudinal crowning for tooth flank on a helical gear surface is usually accomplished by varying the center distance between the hob cutter and work gear, and treated the crossed angle of the hob cutter and work gear as a fixed machine-tool setting. However, in this study, the center distance between the hob cutter and work gear is fixed and the crossed angle is set as a linear function of hob's traverse feed movement for longitudinal crowning tooth flank. Thereby it will induce a twist of tooth flank on the hobbed work that is the reason for a bias of tooth wear and noise of the meshing gear pair. Therefore, to obtain an anti-twist tooth flank of the work gear in longitudinal tooth crowning, a variable pressure angle (VPA) hob cutter is applied during gear hobbing process.

Several researches about hobbing method are recently presented for longitudinal crowning tooth flank of helical gear surfaces. Firstly, a method for longitudinal crowning tooth flank of involute helical gear by varying the center distance was proposed by Litvin et al. [1]. Subsequently, Wang and Fong [2] was proposed a novel face-hobbing method to generate spur gears with longitudinal crowning by two head cutters form an imaginary generating rack with cycloidal lengthwise tooth traces. After that, a mathematical model with a two-parameter equation of meshing for simulation of

${ }^{\mathrm{a} C}$ Corresponding author : yurenwu@ncu.edu.tw 
gear hobbing process was proposed by Chen et al. [3]. Previously, Witte [4] proposed a twist free hobbing solution by analyzing the width of convexity and amount of bias of the tooth flank as well as a finishing hob to cut external crowned helical gears without profile bias. After that, Lange [5] presented some important discussions on the tooth flank's bias errors of helical gears caused in conventional cutting methods and offered techniques used to limit and eliminate bias errors. In the same time, a method for varying the hob profile angle along its lead and shifting the hob diagonally over the whole tooth face width during gear hobbing to reduce the twist of work gear tooth flanks was patented by Winkel [6]. Recently, Hsu and Fong [7] patented the design of a variable tooth thickness (VTT) hob cutter in it's axis and the method of hobbing with a diagonal feed and without varying the center distance. More recently, Tran et al. [8] proposed a novel methodology for finish-hobbing of the twist-free longitudinal crowning tooth flank of helical gears by using a new the hob's diagonal feed motion and a dual-lead hob cutter. And a hobbing method to attain anti-twist tooth flanks of the helical gear in longitudinal tooth crowning by supplementing an additional rotation angle of work gear during its hobbing process is presented by Tran et al. [9]. Finally, the overall geometry, design, and manufacture of a conventional helical gear were detailed in textbook by Litvin and Fuentes [10].

In this research, a mathematical model for anti-twist tooth flank of the work gear surface, hobbed with a new crossed angle and a VPA hob cutter, is constructed in longitudinal crowning process. A numeral example is also presented to illustrate the merits of the proposed gear hobbing method.

\section{Mathematical model of the VPA hob cutter}

The profile of the generating surface of the standard hob cutter is the same as that of the standard helical gear that is generated by a standard rack cutter. To obtain an anti-twist tooth flank of helical gear, the tooth profile of the standard rack cutter is modified with pressure angle changed in it's longitudinal direction, as shown in Fig. 1(a). The generation coordinate systems of the VPA hob cutter are shown in Fig. 1(b), where the coordinate systems $S_{7}, S_{3}$, and $S_{4}$ are rigidly connected to the rack cutter, hob cutter, and frame, respectively. According to Fig. 1, the position vector and unit normal vector of the rack cutter's right-hand side profile can be expressed in coordinate system $S_{3}$ as follows:

$$
\mathbf{r}_{3}=\mathbf{M}_{37} \cdot \mathbf{r}_{7}=\left[\begin{array}{cccc}
\cos \psi_{1} & -\cos \beta_{o 1} \sin \psi_{1} & -\sin \beta_{o 1} \sin \psi_{1} & r_{o 1}\left(\cos \psi_{1}+\psi_{1} \sin \psi_{1}\right) \\
\sin \psi_{1} & \cos \beta_{o 1} \cos \psi_{1} & -\sin \beta_{o 1} \cos \psi_{1} & r_{o 1}\left(\sin \psi_{1}+\psi_{1} \cos \psi_{1}\right) \\
0 & -\sin \beta_{o 1} & \cos \beta_{o 1} & 0 \\
0 & 0 & 0 & 1
\end{array}\right] \cdot\left[\begin{array}{c}
u_{1} \cos \alpha_{o n} \\
-u_{1} \sin \alpha_{o n}+b u_{1} v_{1} \\
v_{1} \\
1
\end{array}\right],
$$

and

$$
\mathbf{n}_{3}=\mathbf{L}_{37} \cdot \mathbf{n}_{7}=\left[\begin{array}{ccc}
\cos \psi_{1} & -\cos \beta_{o 1} \sin \psi_{1} & -\sin \beta_{o 1} \sin \psi_{1} \\
\sin \psi_{1} & \cos \beta_{o 1} \cos \psi_{1} & -\sin \beta_{o 1} \cos \psi_{1} \\
0 & -\sin \beta_{o 1} & \cos \beta_{o 1}
\end{array}\right] \cdot\left[\begin{array}{c}
-b v_{1}+\sin \alpha_{o n} \\
\cos \alpha_{o n} \\
-b u_{1} \cos \alpha_{o n}
\end{array}\right] .
$$

where $u_{1}$ and $v_{1}$ are the surface parameters, $\alpha_{o n}$ is the pressure angle of the rack cutter, and $b$ is VPA coefficient of rack cutter, $\psi_{1}$ is the generated rotation angle of hob cutter around $z_{3}$-axis, and $s_{o n}=r_{o 1} \psi_{1}$ is the rack cutter translates along $z_{4}$-axis and $r_{o 1}$ is the radius of the operating circle of hob cutter, as shown in Fig. 1.

According to the theory of gearing, the equation of meshing between the right-hand side rack cutter surface and left-hand side hob cutter surface can be obtained by

$$
f_{1}\left(u_{1}, v_{1}, \psi_{1}\right)=\mathbf{n}_{3} \cdot \frac{\partial\left[x_{3}\left(u_{1}, v_{1}, \psi_{1}\right), y_{3}\left(u_{1}, v_{1}, \psi_{1}\right), z_{3}\left(u_{1}, v_{1}, \psi_{1}\right)\right]}{\partial \psi_{1}}=0 .
$$


The tooth profile and its unit normal of the VPA hob cutter are defined by considering Eqs. (1), (2) and (3), simultaneously. The locus tooth profile and its unit normal of the standard hob cutter are determined by setting VPA coefficient, $b$, equal zero.

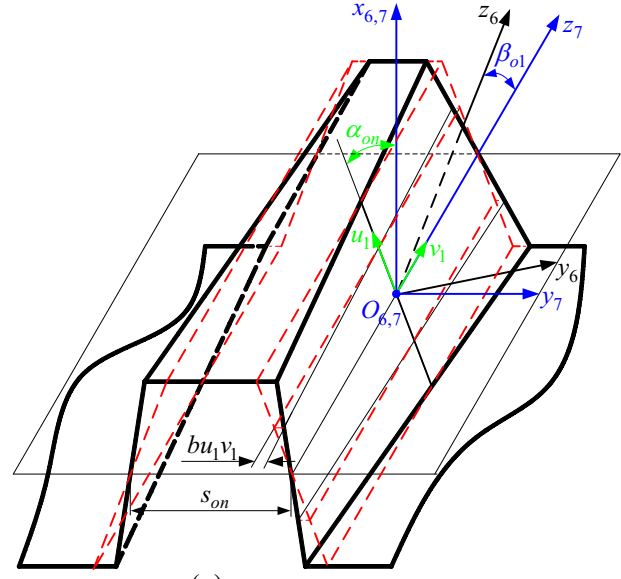

(a)

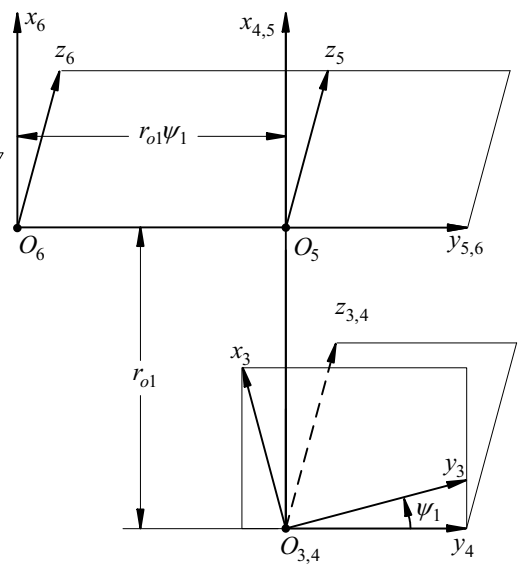

(b)

Figure 1. Surface parameters of VPA rack cutter and generation coordinate systems of VPA hob cutter.

\section{Mathematical model of the longitudinal crowned work gear on gear hobbing machine}

The Coordinate systems for a gear hobbing machine are shown in Fig. 2. Wherein coordinate systems $S_{1}, S_{2}$ and $S_{a}$ are rigidly connected to the hob cutter, work gear and frame of hobbing machine, respectively. While $S_{b}, S_{c}, S_{d}, S_{e}$ and $S_{f}$ are auxiliary coordinate systems for simplification of coordinate transformation. In gear hobbing process, there are two hob's movements: traverse feed movement along the axis of the work gear $z_{a}(t)$, and diagonal feed along the axis of hob cutter $z_{s}(t)=a \cdot z_{a}(t)$. And the crossed angle $\gamma_{o}$ of the hob cutter and work gear is usually fixed as a machine-tool and it can be expressed as follows

$$
\gamma_{o}=\beta_{o 1} \pm \beta_{o 2},
$$

where the $\beta_{o 1}$ and $\beta_{o 2}$ are helix angle of hob cutter and work gear, respectively. The " \pm " sign indicates the same and opposite direction, respectively, of helices between the hob cutter and work gear. for longitudinal crowning tooth flank of work gear, the crossed angle (designated as $\gamma$ ) is modified as a linear function of hob's traverse feed movement in the gear hobbing process as follows:

$$
\gamma=\gamma_{o}+c \cdot z_{a}(t)
$$

where $c$ is crossed angle coefficient between the hob cutter and work gear.

According to Fig. 2, by applying the homogeneous coordinate transformation matrix from coordinate system $S_{3}$ to $S_{2}$, the locus of hob cutter can be represented in coordinate system $S_{2}$ as follows:

$$
\mathbf{r}_{2}\left(u_{1}, v_{1}, \psi_{1}, \phi_{1}, z_{a}(t)\right)=\mathbf{M}_{23}\left(\phi_{1}, z_{a}(t)\right) \cdot \mathbf{r}_{3}\left(u_{1}, v_{1}, \psi_{1}\right)
$$

The rotating angle of work gear $\phi_{2}\left(\phi_{1}, z_{a}(t)\right)$ is set as a linear function of both the hob's rotation angle $\phi_{1}$ and the amount of hob's traverse feed $z_{a}(t)$. This rotational relationship between the hob and work gear is defined as 


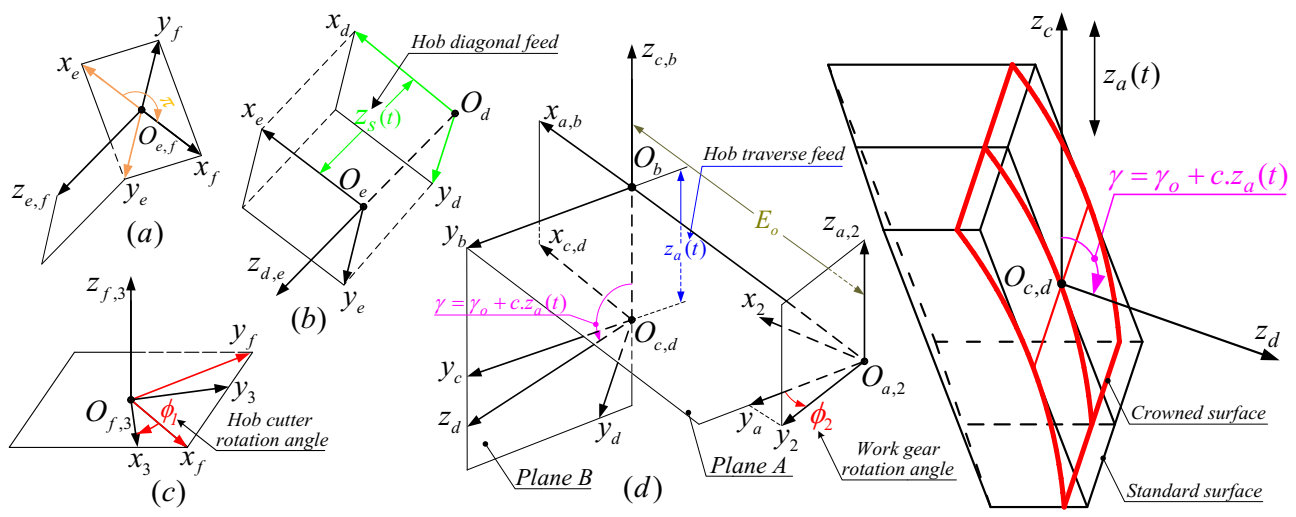

Figure 2. Coordinate systems for a gear hobbing machine.

$$
\phi_{2}\left(\phi_{1}, z_{a}(t)\right)=\frac{N_{1}}{N_{2}} \phi_{1}+\frac{\tan \beta_{o 2}+a \sec \beta_{o 2} \sin \beta_{o 1}}{r_{o 2}} \cdot z_{a}(t),
$$

where symbols $N_{1}$ and $N_{2}$ indicate the number of teeth of the hob cutter and gear, respectively, $r_{o 2}$ is the radius of the operating circle of work gear, and $a$ is the hob diagonal shift coefficient.

Since the gear hobbing process has two independent motion parameters, $\phi_{1}$ and $z_{a}(t)$, there are two equations of meshing between the hob and work gear that can be obtained and expressed as follows:

$$
f_{2}=\mathbf{n}_{2} \cdot \frac{d \phi_{1}}{d t}\left(\frac{\partial\left(x_{2}\left(u_{1}, v_{1}, \psi_{1}, \phi_{1}, z_{a}(t)\right), y_{2}\left(u_{1}, v_{1}, \psi_{1}, \phi_{1}, z_{a}(t)\right), z_{2}\left(u_{1}, v_{1}, \psi_{1}, \phi_{1}, z_{a}(t)\right)\right)}{\partial \phi_{1}}\right)=0,
$$

and $f_{3}=\mathbf{n}_{2} \cdot \frac{d z_{a}(t)}{d t}\left(\frac{\partial\left(x_{2}\left(u_{1}, v_{1}, \psi_{1}, \phi_{1}, z_{a}(t)\right), y_{2}\left(u_{1}, v_{1}, \psi_{1}, \phi_{1}, z_{a}(t)\right), z_{2}\left(u_{1}, v_{1}, \psi_{1}, \phi_{1}, z_{a}(t)\right)\right)}{\partial z_{a}(t)}\right)=0$,

where

$$
\mathbf{n}_{2}\left(u_{1}, v_{1}, \psi_{1}, \phi_{1}, z_{a}(t)\right)=\mathbf{L}_{23}\left(\phi_{1}, z_{a}(t)\right) \cdot\left[n_{x_{3}}\left(u_{1}, v_{1}, \psi_{1}\right), n_{y_{3}}\left(u_{1}, v_{1}, \psi_{1}\right), n_{z_{3}}\left(u_{1}, v_{1}, \psi_{1}\right)\right]^{T}
$$

and $\mathbf{L}_{23}\left(\phi_{1}, z_{a}(t)\right)$ is the submatrix of the transformation matrix $\mathbf{M}_{23}\left(\phi_{1}, z_{a}(t)\right)$ by deleting the last column and row.

The tooth surface of the crowned helical gear can be determined by solving Eqs. (3), (6), (8), (9) and (10), simultaneously.

\section{Numeral example}

The purpose of this example is to validate the proposed longitudinal crowning method. The basic data for work gear, hob cutter and hobbing process are given in Table 1. The level of tooth flank twist of the two double-crowned work gear surfaces is indicated by using two indices: maximum tooth flank twist (designated $M_{c e}$ ) defined by the largest crowning amount subtract the smallest crowning amount and longitudinal crowning evenness ratio (designated $R_{c e}$ ) defined by the smallest crowning amount divided by the largest crowning amount at different position on the edges of work gear surfaces. 
Table 1. Basic data of the gear and hob cutter

\begin{tabular}{|c|c|c|c|}
\hline \multicolumn{2}{|c|}{ Work gear data } \\
\hline \multicolumn{2}{|c|}{ Number of teeth $\left(N_{2}\right)$} & 50 \\
\hline \multicolumn{2}{|c|}{ Normal module $\left(m_{p n}\right)$} & 3 \\
\hline Normal circular-tooth thickness $\left(s_{p n 2}\right)$ & \multicolumn{2}{|c|}{$20^{\circ}$} \\
\hline Normal pressure angle $\left(\alpha_{p n}\right)$ & \multicolumn{2}{|c|}{$20^{\circ}$ R.H. } \\
\hline Helix angle $\left(\beta_{p 2}\right)$ & $18 \mathrm{~mm}$ \\
\hline Face width $\left(F_{\mathrm{w} 2}\right)$ & $120.51 \mathrm{~mm}$ \\
\hline Operating center distance $\left(E_{o}\right)$ & Hobbing process data \\
\hline Hob cutter data & 1 & Original operating crossed angle $\left(\gamma_{o}\right)$ & $107.89^{\circ}$ \\
\hline Number of teeth $\left(N_{1}\right)$ & $87.89^{\circ}$ R.H. & Hob diagonal shift coefficient $(a)$ & -3.26 \\
\hline Helix angle $\left(\beta_{p 1}\right)$ & $4.71 \mathrm{~mm}$ & Crossed angle coefficient $(c)$ & $-1.50 \times 10^{-3}$ \\
\hline Normal circular-tooth thickness $\left(s_{p n 1}\right)$ & & \multicolumn{2}{|c}{} \\
\hline
\end{tabular}

The normal deviations and tooth surface topographies of the crowned helical gear, generated by applying the standard and VPA hob cutters, are calculated and shown in Table 2 and Figs. 3-4, respectively. The maximum tooth flank twist for the crowned work gear tooth flank generated by using the standard hob cutter $(b=0), M_{c e}=19.56 \mu \mathrm{m}$, is much higher than that of the crowned work gear tooth flank generated by using the VPA hob cutter $\left(b=-5.98 \times 10^{-6}\right), M_{c e}=0.87 \mu m$. Besides, the crowning evenness ratio for the tooth flank surface generated by using the standard hob cutter, as shown in Fig. $3\left(R_{c e}=0.19\right)$, is much smaller than that of the tooth flank surface generated by using the VPA hob cutter, as shown Fig. $4\left(R_{c e}=0.94\right)$. It is verified that the twist of the tooth flank almost equals zero by applying the proposed VPA hob cutter.

Table 2. Topography characteristics of tooth flank on the crowned gear

\begin{tabular}{|c|c|c|c|}
\hline Items & VPA coefficient $(b)$ & $\begin{array}{c}\text { Maximum tooth flank twist } \\
\left(M_{c e}(\mu m)\right)\end{array}$ & $\begin{array}{c}\text { Crowning evenness } \\
\text { ratio }\left(R_{c e}\right)\end{array}$ \\
\hline Standard hob cutter & 0 & 19.56 & 0.19 \\
\hline VPA hob cutter & $-5.98 \times 10^{-6}$ & 0.87 & 0.94 \\
\hline
\end{tabular}

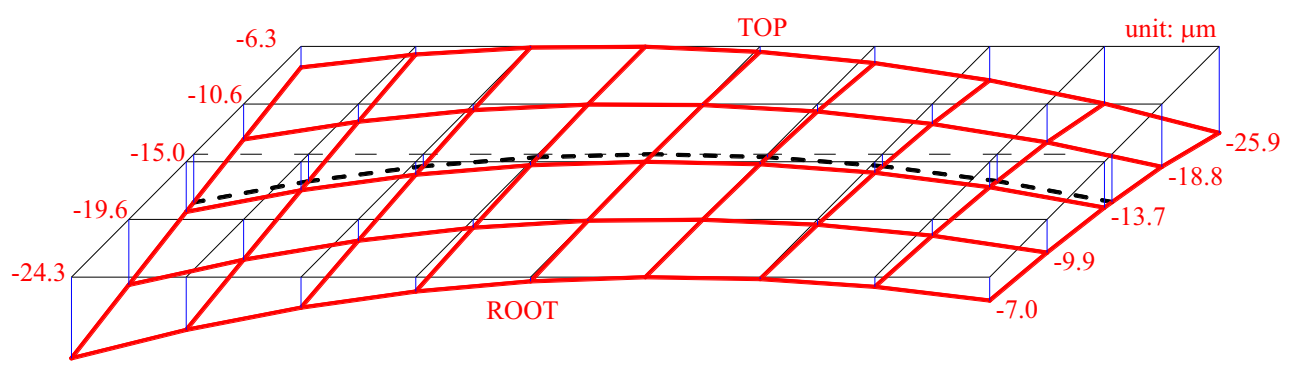

Figure 3. Topography of tooth flank on work gear surfaces hobbed by the standard hob cutter. 


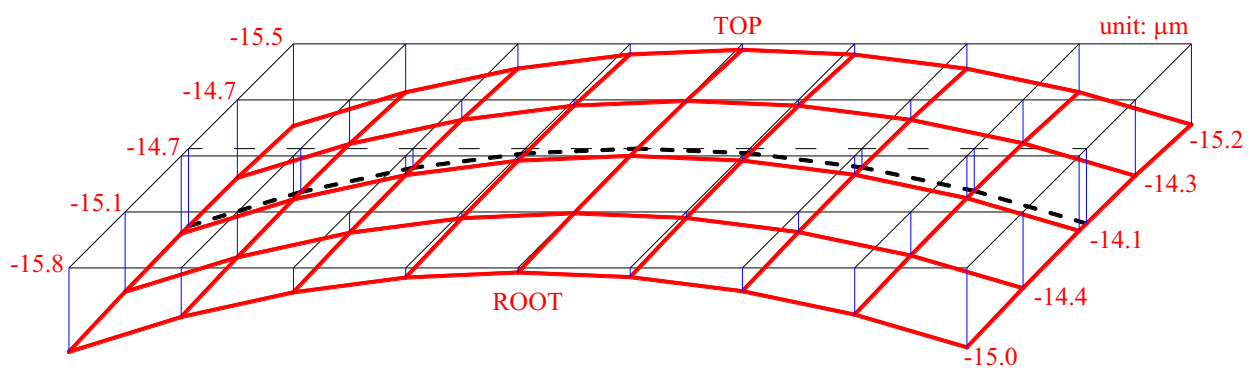

Figure 4. Topography of tooth flank on work gear surfaces hobbed by the VPA hob cutter.

\section{Conclusions}

In this paper, a mathematical model for tooth flank of the crowned work gear surface is constructed in gear hobbing process. According to the simulated results of numeral example, it reveals that the tooth flank of helical gear can be crowned longitudinally by setting the crossed angle between the hob cutter and work gear as a linear function of hob's traverse feed movement and using a standard hob cutter. However, the using of standard hob cutter will induce twist of the tooth flank on the hobbed gear. Therefore, a VPA hob cutter is applied to obtain anti-twist tooth flank. Thereby the twist of tooth flank on crowned work gear is reduced significantly.

\section{Acknowledgments}

The authors are grateful to the Ministry of Science and Technology of the R.O.C for the financial support, contract No: MOST 104-2221-E-035-087- and MOST 104-2221-E-008 -007-.

\section{References}

1. F.L. Litvin, A. Fuentes, I.G. Peres, L. Carvenali, K. Kawasaki, R.F. Handschuh, Modified Involute Helical Gears: Computerized Design, Simulation of Meshing and Stress Analysis, Computer Methods in Applied Mechanics and Engineering 192, 3619-3655 (2003).

2. W.S. Wang, Z.H. Fong, A Dual Face-Hobbing Method for the Cycloidal Crowning of Spur Gears, Mechanism and Machine Theory 43, 1416-1430 (2008).

3. K.H. Chen, C.J. Chen, Z.H. Fong, Computer Simulation for the Cutting Process of a CNC Hobbing Machine, Chinese Society of Mechanism and Machine Theory Conference, Chia-Yi, Taiwan, 154-161 (2009).

4. D. Witte, Finish Hobbing Crowned Helical Gears without Twist, Gear Solutions Magazine, 1213 (2006).

5. J. Lange, How Are You Dealing with the Bias Error in Your Helical Gears, AGMA Fall Technical Meeting (2009).

6. O. Winkel, New Developments in Gear Hobbing, Gear Technology, 47-55 (2010).

7. R.H. Hsu, Z.H. Fong, Novel Variable-tooth-thickness Hob for Longitudinal Crowning in the Gear-hobbing Process, Thirteenth World Congress in Mechanism and Machine Science, Gearing and transmissions, Guanajuato, Mexico (2011).

8. V.T. Tran, R.H. Hsu, C.B. Tsay, A Novel Finish Hobbing Methodology for Longitudinal Crowning of a Helical Gear with Twist-Free Tooth Flanks by Using Dual-Lead Hob Cutters, ASME 2014 International Mechanical Engineering Congress \& Exposition, Montreal, Quebec, Canada. Paper No. IMECE2014-36149 1-9, (2014).

9. V.T. Tran, R.H. Hsu, and C.B. Tsay, Study on the Anti-Twist Helical Gear Tooth Flank With Longitudinal Tooth Crowning, ASME Journal of Mechanical Design 136 (2014). 
ICDES 2016

10. F.L. Litvin, A. Fuentes, Gear Geometry and Applied Theory, second ed., Cambridge University Press (2004). 